(C) 2021 Universidad Nacional Autónoma de México, Facultad de Estudios Superiores Zaragoza.

Este es un artículo Open Access bajo la licencia CC BY-NC-ND (http://creativecommons.org/licenses/by-nc-nd/4.0/).

TIP Revista Especializada en Ciencias Químico-Biológicas, 24: 1-11, 2021.

https://doi.org/10.22201/fesz.23958723e.2021.0.291

\title{
Los isoprenoides como fuente de biocombustibles
}

\author{
${ }^{1}$ Oscar Iván Luqueño-Bocardo, ${ }^{1}$ Juan Pablo Pardo, ${ }^{2}$ Guadalupe Guerra-Sánchez, \\ ${ }^{3}$ James González, ${ }^{1}$ Genaro Matus-Ortega y ${ }^{1 *}$ Lucero Romero-Aguilar \\ ${ }^{1}$ Universidad Nacional Autónoma de México, Facultad de Medicina, Departamento de Bioquímica, Circuito \\ Escolar 411A, Cd. Universitaria, Alcaldía Coyoacán, 04510, Ciudad de México, México. ${ }^{2}$ Instituto Politécnico \\ Nacional, Escuela Nacional de Ciencias Biológicas, Alcaldía Miguel Hidalgo, 11340, Ciudad de México, \\ México. ${ }^{3}$ Facultad de Ciencias, Departamento de Biología Celular, Circuito interior s/n, Cd. Universitaria, \\ Alcaldía Coyoacán, 04510, Ciudad de México, México. E-mail: *lusromaguila@bq.unam.mx
}

\begin{abstract}
RESUMEN
Los combustibles fósiles tienen efectos negativos sobre el medio ambiente y, en los últimos años, la preocupación por el agotamiento de las fuentes de este tipo de energía se ha incrementado, por lo que es necesario la implementación de energías alternas que sean amigables con el medio ambiente, que sean económicas y similares o mejores en cuanto al rendimiento energético que las actuales. Los biocombustibles son una fuente de energía que se obtienen de los lípidos de plantas como el maíz, o de los lípidos de microorganismos como las cianobacterias, las bacterias y las levaduras oleaginosas como Rhodosporidium toruloides y Yarrowia lipolytica, estas últimas con una capacidad de acumular hasta el 70\% de lípidos en relación a su peso seco. $R$. toruloides también tiene la particularidad de acumular carotenoides, un tipo de terpeno que tiene importancia comercial. Los biocombustibles que se obtienen de las levaduras, por su origen, se clasifican como de tercera generación, y son energética y estructuralmente similares a los que se extraen de los fósiles, por lo que algunos isoprenoides pueden ser utilizados en la industria de la aviación y automotiz para motores diesel.

$R$. toruloides tiene la ventaja de integrar a su metabolismo una amplia variedad de azúcares: glucosa, xilosa, manosa, sacarosa, mientras que $S$. cerevisiae y $Y$. lipolytica son incapaces de asimilar la xilosa. $R$. toruloides también tolera variaciones en la temperatura y el $\mathrm{pH}$. Después de $Y$. lipolytica, $R$. toruloides es una de las levaduras más exploradas para la producción de biomoléculas, como los lípidos y los terpenos, a partir de los cuales es posible obtener combustibles y aditivos de combustibles. En esta revisión nos enfocamos en describir algunas propiedades de los isoprenoides y sus aplicaciones como combustibles y aditivos de combustible.
\end{abstract}

Palabras clave: monoterpenos, lípidos, R. toruloides, biocombustibles, sesquiterpenos, Y. lipolytica.

The isoprenoids as biofuel source

\begin{abstract}
The fossil fuels have negative effects on the environment thus in last years the concern about the supplies of petroleum has increased, therefore the implementation of renewable energies is a necessity, these energies also have to be economic and environment-friendly. The biofuels, are environment-friendly energy that can be obtained from renewable sources as corn, lignocellulose, citrus peel, or from the lipids of microorganism as cyanobacteria, genetically modified bacteria Escherichia coli and yeast as Yarrowia lipolytica and Rhodosporidium toruloides. Both yeasts have the capacity to accumulate $70 \%$ of lipid in dry biomass, unlike Y. lipolytica, the $R$. toruloides accumulate carotenoids, a kind of terpenoid with many applications in pharmaceutics and food industries. Moreover, this yeast has the ability to metabolize a variety of sugars as glucose, xylose, mannose, and sucrose, while the yeasts Y. lipolytic and Saccharomyces cerevisiae are unable to metabolize the pentose xylose. The $R$. toruloides also can tolerate variations in temperature and $\mathrm{pH}$ reason why is one of the yeast more explored to achieve the production of biomolecules as lipids and terpenoids, from which is possible to obtain biofuels and biofuels additives. The biofuels obtained from yeast, due to its origin, are classified as biofuels of third generation, and energetic and structurally are similar to fossil fuels, therefore some isoprenoids are used in the aviation industry and can be used for diesel engines. In this review, we describe some properties of the isoprenoids as precursors of biofuels and additives.
\end{abstract}

Keywords: monoterpenes, lipids, $R$. toruloides, biofuels, sesquiterpenes, Y. lipolytica.

Artículo recibido el 30 de septiembre del 2020.

Artículo aceptado el 01 de febrero del 2021. 


\section{INTRODUCCIÓN}

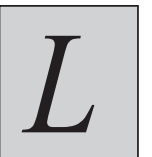

as levaduras son un modelo de estudio de la célula eucariota por excelencia. En los últimos años, estos microorganismos han ganado relevancia en el campo de la biotecnología. Actualmente, sabemos que el esponjado de un pan o la obtención de una bebida como la cerveza y el vino son el resultado de un proceso de fermentación realizado por la levadura Saccharomyces cerevisiae. Sin embargo, tanto en $S$. cerevisiae como en otras levaduras el proceso de la degradación de la glucosa y su posterior conversión a $\mathrm{CO}_{2} \mathrm{y}$ etanol no son los únicos productos de importancia biotecnológica en las levaduras.

Así, a través de múltiples fases de experimentación y análisis a lo largo de los años, se han descubierto y descrito otras levaduras capaces de producir biomoléculas como los isoprenoides o los terpenos simples y complejos, que son precursores de componentes celulares importantes como el ergosterol y el colesterol, moléculas que se sintetizan a partir del farnesil pirofosfato (FPP), y que son parte de la estructura de las membranas celulares en levaduras y en mamíferos, respectivamente (Ericsson, Appelkvist, Runquist \& Dallner, 1993). El colesterol también es importante para la síntesis de ácidos biliares, vitamina D y hormonas esteroideas. Mientras que el ergosterol es precursor de la vitamina D2, que se utiliza como aditivo en alimentos, así como de otros fármacos esteroideos: cortisona y progesterona. El ergosterol en los hongos es esencial en el proceso de adaptación al estrés causado por los procesos de fermentación, sin embargo, la baja disponibilidad de oxígeno afecta negativamente la biosíntesis del ergosterol (Hu et al., 2017). Los isoprenoides participan en procesos como la respiración celular (ubiquinona) y el transporte de oligosacáridos en el retículo endoplásmico (dolicol), así como en la prenilación de las proteínas.

Los isoprenoides se construyen a partir del isopentinil difosfato (IPP) y el dimetilalilpirofosfato (DMAPP), que se encuentran básicamente en los tres dominios (archaea, bacteria y eukarya) (Chang, Song, Liu \& Liu, 2013). Los terpenos y sus derivados, los terpenoides, son responsables del color y aroma en plantas, por ejemplo, el color naranja de las zanahorias y de la flor de cempasúchil se debe a la síntesis y acumulación de carotenoides. El aroma de los cítricos, del eucalipto y del pino también son el resultado de la síntesis de terpenos (Langi, Kiokias, Varzakas \& Proestos, 2018; Tetali, 2019). Actualmente, el uso y las aplicaciones de los terpenoides no sólo se limitan a proporcionar color y aroma, sino que también son precursores de fármacos como la artemisina (amorfadieno), usada para tratar la malaria, del bisaboleno y el farneseno, como precursores de biocombustibles (Tetali, 2019; Yaegashi et al., 2017). Para la síntesis de isoprenoides y lípidos es necesario la acetil-CoA. En años recientes, los isoprenoides han adquirido importancia como posibles fuentes renovables y ecológicas de combustible (Das, Patra \& Ghosh, 2020).

Los isoprenoides se clasifican, según el número de átomos de carbono, como hemiterpenos (C5), monoterpenos (C10), sesquiterpenos (C15), diterpenos (C20), triterpenos (C30), tetraterpenos, politerpenos y meroterpenos (> C40). De estos grupos, el de los monoterpernos, de levaduras como $S$. cerevisiae (ascomycota) o Rhodosporidium toruloides (basidiomycota), es sobre los que se han hecho más estudios para su producción a través de la ingeniería genética. Esto se debe a sus características fisicoquímicas "compatibles" con las de los combustibles derivados del petróleo: el combustible fósil (diesel D2) utilizado en motores de encendido por comprensión, que es una mezcla de alcanos lineales, ramificados y cíclicos, con una longitud promedio de 16 átomos de carbono (George, Alonso-Gutierrez, Keasling \& Lee, 2015). Comparado con los combustibles fósiles, el biodiesel que se obtiene de microorganismos como las levaduras ofrece una reducción de las emisiones contaminantes: $\mathrm{CO}$ de $48 \%$, $\mathrm{SO}_{2}$ de $100 \%, \mathrm{CO}_{2}$ de $67 \%$ y partículas pequeñas de $47 \%$. Además de su naturaleza no tóxica, este biodiesel se clasifica como de tercera generación debido a que no se obtiene de plantas y semillas (Singh, Jeyaseelan, Bandyopadhyay \& Paul, 2018).

\section{Conectando con el metabolismo}

Aunque las modificaciones genéticas han sido exitosas, la ingeniería genética aún se enfrenta con problemas metabólicos propios de la célula que disminuyen la producción de biomoléculas de interés comercial, dificultades que hoy en día no se han resuelto. Aunque los modelos convencionales como $S$. cerevisiae y Escherichia coli son fáciles de modificar, presentan limitaciones para utilizar a la lignocelulosa como fuente de carbono, debido a que no tienen la maquinaria necesaria para degradar a la lignocelulosa y asimilar a la xilosa, uno de los componentes más abundante de la lignocelulosa. La asimilación de la xilosa se realiza por la xilosa reductasa que toma a la xilosa y la transforma en xilitol, el cual, es oxidado por la xilitol deshidrogenasa a xilulosa, que al ser fosforilada por la xilulocinasa produce la xilulosa-5-fosfato, que se metaboliza en la vía de las pentosas fosfato. $S$. cerevisiae es capaz de crecer en xilulosa pero no en xilosa, y sólo utiliza a esta última en presencia de glucosa cuando se sobreexpresan los genes de una aldosa reductasa inespecífica, GRE3, y de la xilitol deshidrogenasa, ScXYL2 (Toivari, Salusjarvi, Ruohonen \& Penttila, 2004). Para que $S$. cerevisiae sea capaz de asimilar xilosa, la vía del catabolismo de la xilosa se ha insertado en el genoma de la levadura utilizando el sistema CRISPR/cas9, obteniéndose como producto el alcohol primario butanodiol. La deshidrogenación del butanodiol produce saborizantes, combustible, resinas, pinturas y solventes. Su hidrogenación genera octano, combustible de alta calidad para la aviación (Das et al., 2020; Hakizimana, Matabaro \& Lee, 2020). 
En $E$. coli se ha explorado la vía de Weimberg para lograr la conversión de xilulosa a 2-oxoglutarato (alfa-cetoglutarato), intermediario del ciclo de Krebs, sin la pérdida de átomos de carbono en forma de $\mathrm{CO}_{2}$, que ocurre cuando se produce el 2-oxoglutarato a partir de la xilulosa-5-fosfato o el piruvato a partir del 2-ceto-3 desoxi-D-xilonato (vía de Damh), la cual al entrar a la vía de las pentosas fosfato produce el gliceraldehído3-fosfato, un intermediario de la glucólisis (Figura 1) (Rossoni et al., 2018). Aunque esta es una cepa prometedora, quedan por resolver los efectos de los subproductos de la degradación de la lignocelulosa.

Otro reto de la bioingeniería es la inserción de los genes que codifican para las moléculas de interés y que éstas no resulten tóxicas para el microorganismo. En $S$. cerevisiae se ha logrado la inserción de la bisaboleno monoterpeno sintasa y la cineol sintasa, que participan en la síntesis de terpenos que se pueden utilizar como biocombustible. De manera natural, la levadura tiene a la sintasa bifuncional farnesil pirofosfato sintasa, codificada por el gen Erg20, la cual tiene actividad de geranil pirofosfato y farnesil pirofosfato sintasa. A partir del isopentenil pirofosfato $\mathrm{y}$ del dimetilalil pirofosfato, esta enzima sintetiza el geranil pirofosfato (GPP, C10), pero casi no lo libera al medio, sino que lo utiliza para la síntesis del farnesil pirofosfato. Debido a esto, la poza de GPP es pequeña, lo cual afecta la síntesis del monoterpeno cineol y la del sesquiterpeno bisaboleno, que al ser hidrogenado genera el bisabolano, el cual tiene propiedades de biocombustible (Figura 2). Los sesquiterpenos son el resultado de la condensación de dos moléculas de IPP con una de DMAPP, el incremento en la síntesis de sesquiterpenos se ha intentado en E.coli y $S$. cerevisiae a través de la sobreexpresión de la farnesil pirofosfato sintasa o la bisabolano sintasa (Das et al., 2020). Sin embargo, la poza de GPP es clave para la producción de estos terpenos, ya que si ésta es pequeña, no se incrementa la producción de los terpenos (Figura 3). Para incrementar el flujo se han creado mutantes como la ERG20 k197G, que expresa a la geraniol sintasa de Ocimum basilicum y puede producir $5 \mathrm{mg} / \mathrm{L}$ de geraniol (Zhao et al., 2017).

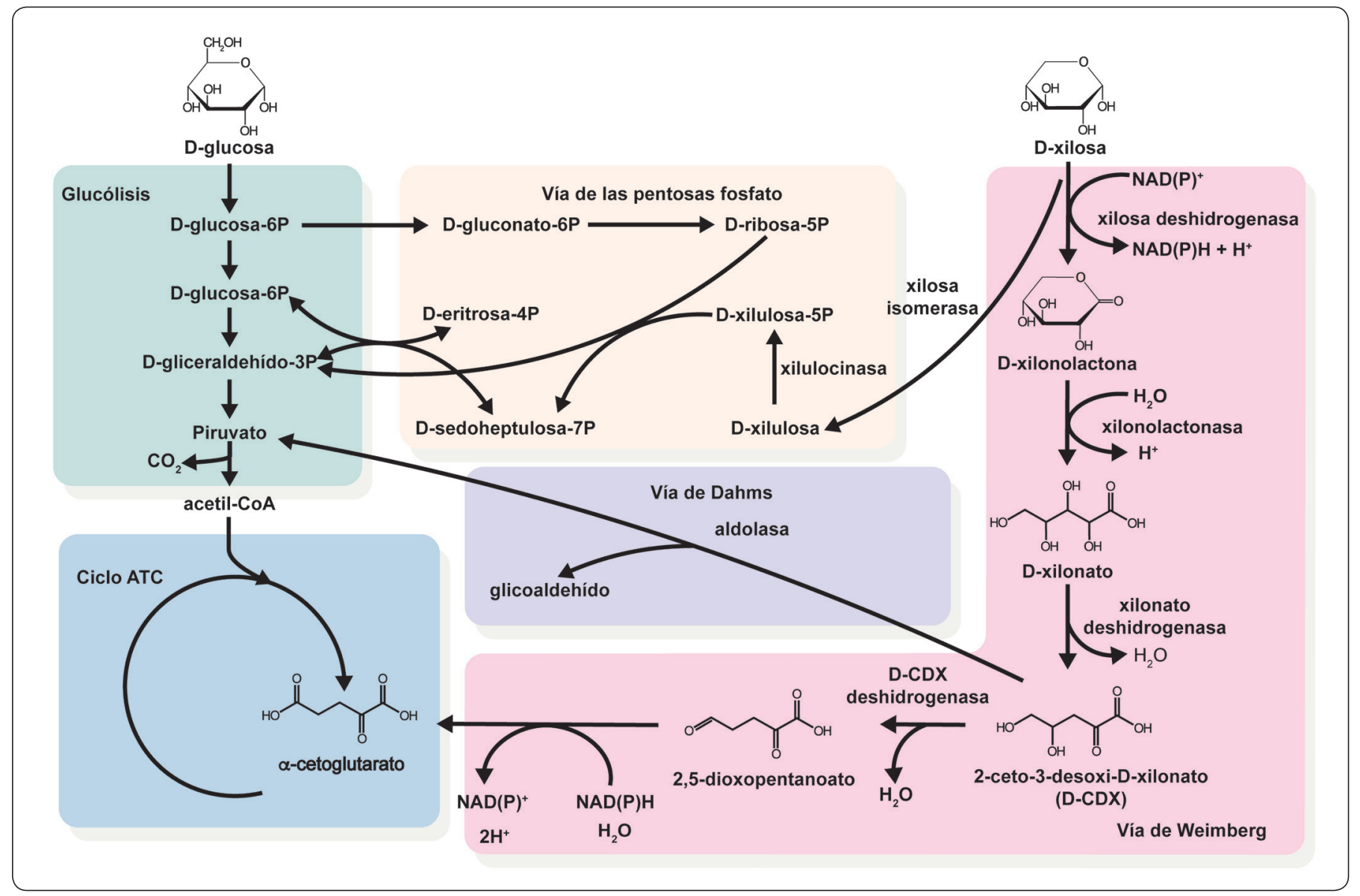

Figura 1. Asimilación de la D-xilosa a través de las vías de las pentosas fosfato, Weimberg y Dahms. Las reacciones de las vías de las pentosas fosfato y Dahms son nativas de $E$. coli. Las cinco reacciones de la vía de Weimberg (color naranja) requieren enzimas de Caulobacter crescentus. La hidrólisis de la xilonolactona puede ocurrir espontáneamente y, en $E$. coli, la deshidratación del xilonato es catalizada por la actividad de yjhG/yagF (familia de D-xinolato deshidratasa). Modificada de (Rossoni et al., 2018). 


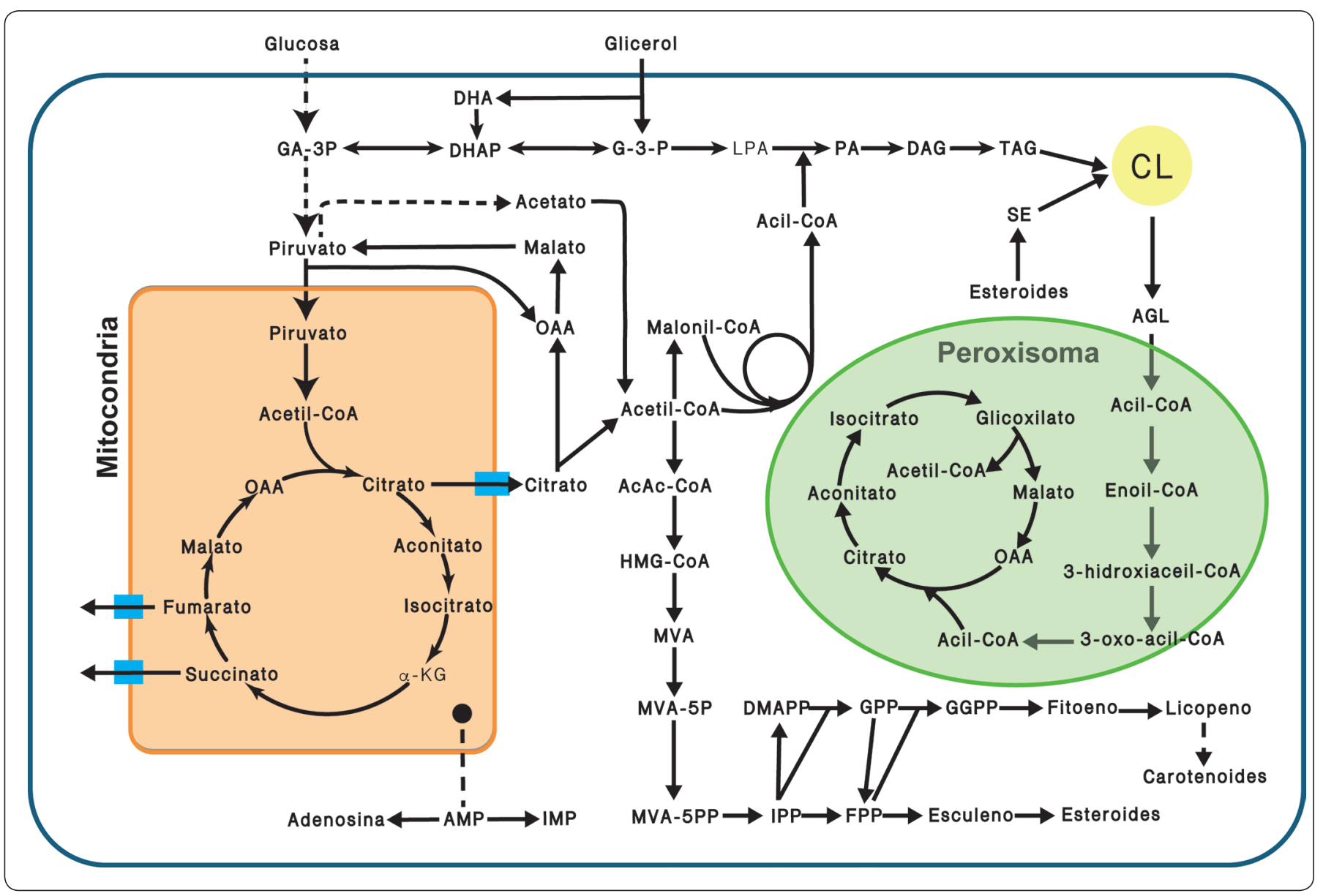

Figura 2. Vías metabólicas de $\boldsymbol{R}$. toruloides. Glucólisis, ciclo de Krebs en la mitocondria, biosíntesis y degradación de ácidos grasos, triacilgliceroles y fosfolípidos, biosíntesis de isoprenoides. Abreviaturas: GA-3P, gliceraldehído-3-fosfato; DHA, dihidroxiacetona; DHAP, dihidroxiacetona fosfato; G3P, glicerol-3-fosfato; LPA, ácido lisofosfatídico; PA, ácido fosfatídico; DAG, diacilglicerol; TAG, triacilglicerol; SE, ésteres de esterol; AGL, ácidos grasos libres; $\mathrm{CL}$, cuerpos lipídicos; OAA, oxaloacetato; $\alpha$-KG, $\alpha$-cetoglutarato; AcAcCoA, aceto-acetil-CoA; HMG-CoA, hidroximetilglutaril-CoA; MVA, mevalonato; MVA-5P, mevalonato-5-fosfato; MVA-5PP, mevalonato-5-difosfato; IPP, isopentenil difosfato; DMAPP, dimetilalil pirofosfato; GPP, geranil pirofosfato; FPP, farnesil pirofosfato, GGPP, geranilgeranil pirofosfato; AMP, adenosín monofosfato; IMP, inosina monofosfato. Modificada de (Wen et al., 2020).

Por otra parte, debido a su naturaleza lipofílica, los terpenos son tóxicos para las células: el monoterpeno $\beta$-pineno incrementa la fluidez de la membrana mitocondrial, inhibe la respiración y en consecuencia la síntesis de ATP; además, inhibe el transporte de $\mathrm{K}^{+} \mathrm{e}^{+}$a través de la membrana plasmática.

\section{Algunos terpenos de importancia comercial}

El limoneno es un monoterpeno cíclico producto de la degradación enzimática de la cáscara de naranja o limón. Este monoterpeno se ha explorado como combustible para aviones y como aditivo de combustibles para mejorar el rendimiento en climas fríos (Zhang \& Zhao, 2015); sin embargo, es tóxico para los microorganismos. La concentración mínima inhibitoria para el crecimiento de $S$. cerevisiae es de $0.44 \mathrm{mM}$, por lo que se ha propuesto el uso de un sistema de extracción por solventes para retirar al monoterpeno e incrementar la tolerancia de esta levadura y lograr una mayor producción (Brennan, Turner, Kromer \& Nielsen, 2012), un proceso que aumenta los costos de producción, pero que evita la inhibición de la producción de bioetanol y biogás (Pourbafrani, Forgacs, Horvath, Niklasson \& Taherzadeh, 2010). También es un agente quimioterapéutico, induce el incremento de los factores pro-apoptóticos y disminuye los anti-apoptóticos, inhibe la isoprenilación de las proteínas $G$ pequeñas, a las ATPasas $\mathrm{Na}^{+} / \mathrm{K}^{+}$e induce a los proto-oncogenes (Mukhtar, Adu-Frimpong, Xu \& Yu, 2018).

El farneseno (C15), al igual que el limoneno, requiere de una hidrogenación química para saturar sus dobles enlaces y producir combustibles alcanos. La forma reducida del farneseno es el farnesano, el cual se usa como 


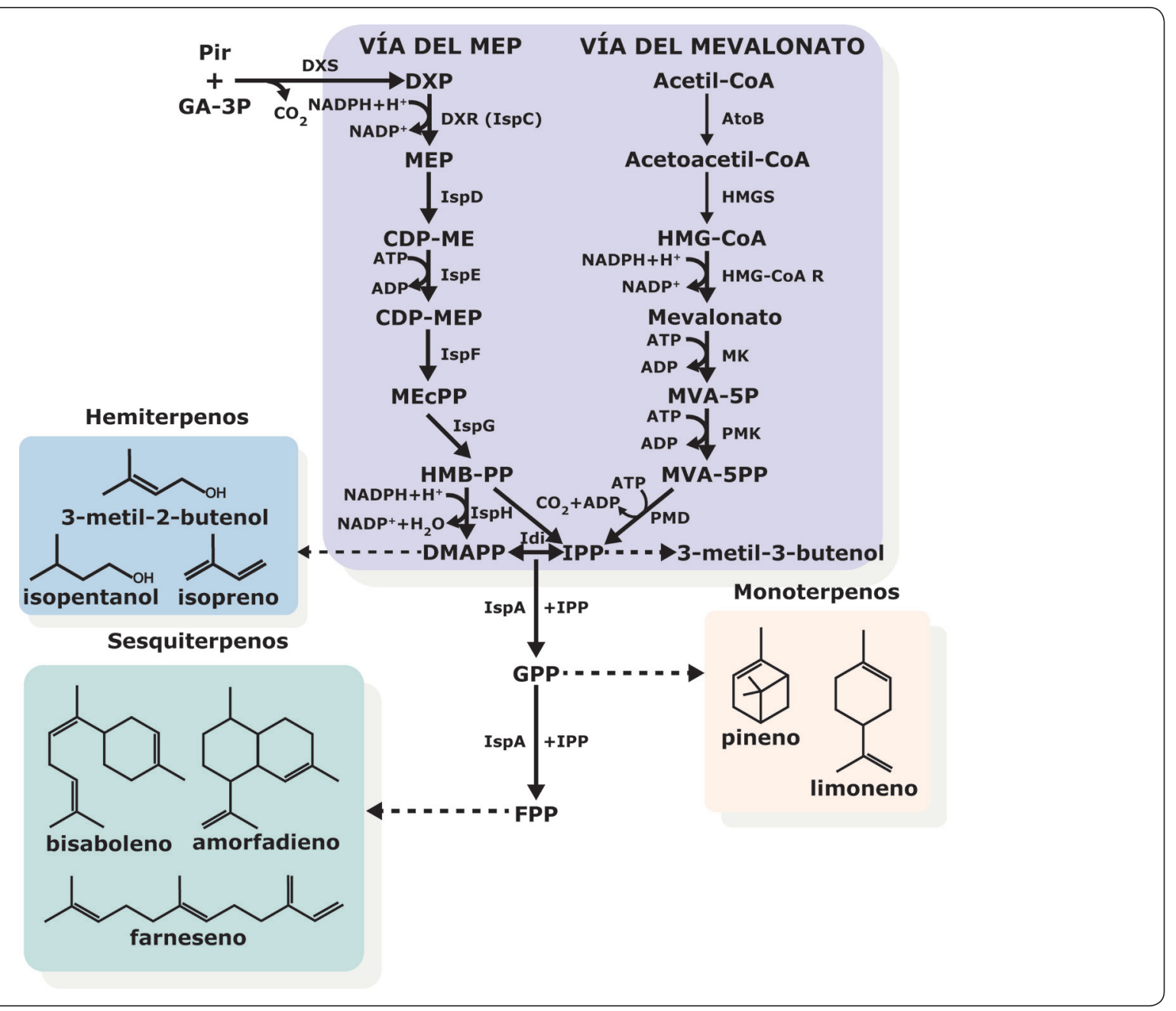

Figura 3. Biosíntesis de isoprenoides. La vía del MEP/MVA produce los intermediarios DMAPP e IPP. EI DMAPP, es el precursor de los isoprenos e isopentanol. La condensación del DMAPP e IPP produce el GPP y el FPP, precursores de los monoterpenos (pineno y limoneno) y los sesquiterpenos (bisabolano, amorfadieno y farneseno), respectivamente. Abreviaturas: DXP, 1-desoxi-D-xilulosa 5-fosfato; DXS, DXP sintasa; DXR, DXP reductoisomerasa; MEP, 2-C-metil-D-eritritol 4-fosfato; IspD (-)-trans-isopiperitenol deshidrogenasa; CDP-ME, 4-difosfocitidil-2-C-metil-D-eritritol; MEcPP, 2-C-metil-D-eritritol 2,4-ciclodifosfato; IspF, MEcPP sintasa; HMB-PP, hidroximetilbutenil pirofosfato; IspG, HMB-PP sintasa; IspH, HMB-PP reductasa; dimetilalil pirofosfato; AtoB, acetoacetilCoA tiolasa; HMG-S, HMG-CoA sintasa; HMG-R, HMG-CoA reductasa; MK, mevalonato cinasa; MVA-5P, mevalonato 5-fosfato; PMK, fosfomevalonato cinasa; MVA-5PP, mevalonato 5-pirofosfato; PMD, fosfomevalonato descarboxilasa; IPP, isopentinil pirofosfato; FPP, farnesil pirofosfato; IspA, FPP sintasa; GPP, geranil pirofosfato. Modificada de (Li et al., 2020).

biocombustible. El farneseno, un sesquiterpeno que deriva del FPP, lo produce la farneseno sintasa y comúnmente se usa como componente de fragancias y saborizantes (Figura 3). En la naturaleza es un atrayente para la polinización y una alarma de feromonas de insectos. Con la expresión de la vía del mevalonato en $E$. coli se ha alcanzado una producción de farneseno de $728 \mathrm{mg} / \mathrm{L}$ a las $72 \mathrm{~h}$ y por arriba de $1.1 \mathrm{~g} / \mathrm{L}$ después de las $120 \mathrm{hr}$ de incubación. En S. cerevisiae (PE-2), utilizando cepas modificadas, se ha alcanzado una producción de farneseno mayor al $50 \%$ del rendimiento de masa teórica (estimación de la producción máxima posible de un producto que puede obtenerse en una reacción química). El farnesano tiene un número de cetano (calidad de ignición) de 58, un punto de nube (temperatura de formación de cristales) de $-78^{\circ} \mathrm{C}$, el del diesel D2 es de $-3{ }^{\circ} \mathrm{C}$. La densidad del farnesano es $0.77 \mathrm{~g} / \mathrm{mL}$, más baja que la del bisabolano (George et al. 2015; Gupta \& Phulara 2015). Estos valores se comparan con los del diesel, número de cetano 51 mínimo, índice de cetano 46 mínimo, densidad $820 \mathrm{Kg} / \mathrm{m}^{3}$ mínimo, punto de ignición $>55{ }^{\circ} \mathrm{C}$ (https:// www.chevron.com/-/media/chevron/operations/documents/ diesel-fuel-tech-review.pdf). 
La producción de bisabolano por $E$. coli y $S$. cerevisiae se ha realizado a través de la inserción de sintasas de bisaboleno provenientes de plantas. La bisaboleno sintasa de Abies grandis es la más prometedora, y junto con la ingeniería del flujo de FPP, se han producido hasta $900 \mathrm{mg} / \mathrm{L}$ del terpeno en ambos microorganismos. El bisabolano (D2 diesel) tiene un número de cetano de 52 , un punto de nube de -25 , una densidad de $850 \mathrm{Kg} / \mathrm{m}^{3}$, y un punto de inflamabilidad de 60-80 ${ }^{\circ} \mathrm{C}$ (George et al., 2015). Los alcoholes de cadena corta (metanol, butanol, propanol, etanol) también pueden ser esterificados para obtener biocombustibles (Salis, Pinna, Monduzzi \& Solinas, 2005), ya sea por biocatálisis, utilizando lipasas, o por catálisis alcalina, ambos procesos costosos.

El farnesol es un alcohol sesquiterpenoide lineal derivado de la desfosforilación del FPP (Figura 3). Farmacéuticamente es relevante como antimicrobiano, precursor de fármacos anticáncer, biopesticida y biocombustible. En E. coli la desfosforilación del FPP se realiza por la fosfatasa endógena NudB, que tiene la capacidad de tomar al IPP/DMAPP y transformarlos en isopentenol. El farnesol se ha producido en $S$. cerevisiae en una cepa con la escualeno sintasa regulada a la baja, produciéndose $80 \mathrm{mg} / \mathrm{L}$, mientras que con la sobreexpresión de la hidroximetilglutaril-CoA reductasa (HMG-CoA R) se genera $145.7 \mathrm{mg} / \mathrm{L}$. En E. coli se producen $135 \mathrm{mg} / \mathrm{L}$. La ingeniería de una de las fosfatasas endógenas de E.coli podría aumentar la producción del farnesol (Gupta \& Phulara, 2015; Zada et al., 2018).

\section{Levaduras productoras de biocombustible}

En el campo de la producción de biocombustibles también se emplean otras levaduras como Yarrowia lipolytica $y$ Rhodosporidium toruloides.

La levadura oleaginosa $Y$. lipolytica, es capaz de producir hasta el $80 \%$ de su peso seco en lípidos, los cuales pueden ser usados para la obtención de biocombustibles debido a su parecido con los aceites vegetales que, por medio de reacciones de transesterificación, se convierten en biodiesel del tipo D2, utilizado en camiones de carga, trenes, vehículos, entre otros (Xu, Qiao, Ahn \& Stephanopoulos, 2016). La transesterificación es el reemplazo del grupo alcohol de un éster por otro alcohol, como el etanol, metanol, propanol, butanol, isopropanol, ter-butanol y octanol. Esta reacción se lleva a cabo en la presencia de catalizadores básicos homogéneos como el $\mathrm{NaOH}$ o $\mathrm{KOH}$, carbonatos u óxidos. El metanol genera ésteres metílicos de ácidos grasos (FAMES) más volátiles, sin embargo, el metanol es más tóxico. Los ésteres etílicos de ácidos grasos (FAEE) son más viscosos, presentan puntos de nube y fluidez más bajos que los FAMES (Yusoff, Xu \& Guo, 2014).
Otro bio-producto derivado de los lípidos ricos en ácidos grasos insaturados es el ácido linoleico (omega-6), que se usa como suplemento alimenticio. Este ácido se produce en condiciones en donde la $\beta$-oxidación y la capacidad de almacenar triacilgliceroles (TAG) están suprimidas y el gen de la $\Delta 12$-desaturasa se sobreexpresa, produciendo $320 \mathrm{mg} / \mathrm{L}$ del ácido linoleico, que debido a su toxicidad debe de exportarse al medio extracelular. Las cepas incapaces de degradar lípidos vía $\beta$-oxidación o almacenarlos en forma de TAG son más sensibles a los ácidos grasos libres (Imatoukene et al., 2017). Otros genes del metabolismo de los lípidos que se han modificado con gran éxito son: 1) la sobreexpresión del gen de la glicerol-3-fosfato deshidrogenasa (GPD1) junto con la eliminación de su isoforma GUT2, en una cepa con la $\beta$-oxidación truncada, se duplica la producción de lípidos; 2) la sobreexpresión del gen de la acetil-CoA carboxilasa $(A C C 1)$ junto con la eliminación del gen de la diacilglicerol aciltransferasa $(D G A 1)$ o la sobreexpresión del gen de la $D G A 1$ y la eliminación del gen del factor de la biogénesis peroxisomal 10 (pex10) y del gen de la hidroxiacil-coenzima A deshidrogenasa peroxisomal ( $m f e l)$, se aumenta la producción de lípidos hasta cinco veces comparada con la de la cepa silvestre (Gajdos, Ledesma-Amaro, Nicaud, Certik \& Rossignol, 2016; Niehus, Crutz-Le Coq, Sandoval, Nicaud \& Ledesma-Amaro, 2018). En esta levadura también se produce el ácido ricinoleico, que se sintetiza en una cepa con la $\beta$-oxidación truncada y con la $\Delta 12$-hidroxilasa de ácidos grasos (RcFAH12) sobreexpresada, logrando un aumento en la producción de lípidos de hasta un $43 \%$ (Beopoulos et al., 2014).

La cepa silvestre de $Y$. lipolytica es incapaz de incorporar la xilosa a su metabolismo, pero la cepa $Y$. lipolityca- Pold, que expresa heterólogamente la xilosa reductasa y la xilitol deshidrogenasa, junto con la sobreexpresión de la xilulosa cinasa nativa, asimila la xilulosa de residuos de agave como única fuente de carbono y la dirige a la acumulación de lípidos (Niehus et al., 2018). Esta levadura es una de las más estudiadas en cuanto a la producción de lípidos y sus diferentes aplicaciones, pero no es la única, existen otros microorganimos con características metabólicas prometedoras como el basidiomiceto Rhodosporidium toruloides, una levadura oleaginosa roja, perteneciente al subphylum Pucciniomycotina, que se aisló de la pulpa de madera, aunque también puede encontrarse en el suelo, el agua de mar y en aguas residuales así como en las hojas de las plantas. Es una levadura con la capacidad de acumular más del 76\% de lípidos en relación a su peso seco. Las limitaciones de fosfato y sulfato favorecen la acumulación de lípidos en $R$. toruloides (Dinh et al., 2019; Zhao, Peng, Du, Liu \& Liu, 2012). Además, de manera natural produce carotenoides, los cuales se derivan del acetil-CoA y tienen un alto potencial 
como biodiesel. La capacidad de producir carotenoides es una característica valiosa para la biotecnología, debido a que no todas las levaduras son capaces de producirlos. Los carotenoides son moléculas que protegen contra la radiación ultravioleta y el estrés oxidativo (Liu et al., 2018). Actualmente, $R$. toruloides es un modelo para la producción de monoterpenos con potencial uso como biocombustibles, principalmente el jet fuel, a través de la inserción en su genoma de diferentes monoterpeno sintasas provenientes de plantas y otros hongos. Es una levadura con amplia capacidad metabólica. Como fuente de carbono puede asimilar hexosas, pentosas, ácidos orgánicos (D-galacturónico), etanol, glicerol y generar grandes cantidades de biomasa ( $100 \mathrm{~g} / \mathrm{L}$ masa en peso seco) (Wen, Zhang, Odoh, Jin \& Zhao, 2020; Zhao et al., 2012). Estas características son relevantes para la bioingeniería, que en los últimos años ha hecho un gran esfuerzo para producir biocombustibles económicos a partir de fuentes de carbono de fácil obtención y poco contaminantes, como el maíz o la lignocelulosa.

$R$. toruloides cultivada en cáscara de naranja, pera, mango papaya, coliflor o repollo, como fuente de carbono, produce un $49 \%$ en peso seco de lípidos saturados: mirístico, palmítico, esteárico, lignocérico y cerótico y $50 \%$ en peso seco de lípidos insaturados; oleico, margárico, linoleico (omega-6) y nonadecanoico. Estos ácidos grasos, por transesterificación ácida, se convierten en biodiesel con una tasa de conversión de hasta el 35.6\% (Singh et al., 2018).

La lignocelulosa, un componente de la pared celular de las plantas, está formada por celulosa, hemicelulosa y lignina. Se ha propuesto a la lignocelulosa como materia prima para la producción de los biocombustibles: gasolina, diesel, jet fuel y bioetanol. Aunque la lignocelulosa es una fuente de carbono de rápido acceso, lo cual la hace económicamente atractiva, tiene como desventaja que para poder ser utilizada es necesario un proceso de hidrólisis que libere a los azúcares fermentables: glucosa, xilosa, arabinosa, manosa y galactosa (de Paula et al., 2019). Los procesos de hidrólisis pueden consistir en tratamientos con celulasas, hemicelulasas, reacciones de oxidación catalizadas por peroxidasas y lacasas, tratamientos alcalinos o por sistema de iones líquidos. La hidrólisis y la degradación de las pentosas de la lignocelulosa generan alcoholes, aldehídos (furfural) y la lignina, que tiene como principal componente al ácido $p$-cumárico (Rodriguez et al., 2019). Estos compuestos son tóxicos para la mayoría de las levaduras, como $S$. cerevisiae y $Y$. lipolityca, mientras que $R$. toruloides parece tolerar algunos de estos subproductos: el crecimiento de la levadura se ve afectado por concentraciones de $6 \mathrm{~g} / \mathrm{L}$ de ácido fórmico, mientras que con $2 \mathrm{~g} / \mathrm{L}$ hay generación de biomasa siempre que los otros compuestos tóxicos estén a bajas concentraciones (Yaegashi et al., 2017; Zhao et al., 2012). S. cerevisiae tolera $<5 \mathrm{~g} / \mathrm{L}$ de ácido acético y un $\mathrm{pH}$ de 4.5 externo, $R$. toruloides tolera hasta $10 \mathrm{~g} / \mathrm{L}$ del mismo ácido en presencia de glucosa y arabinosa, mientras que, si sólo está presente el ácido en el medio, la levadura solamente tolera hasta $5 \mathrm{~g} / \mathrm{L}$; en presencia de glucosa, xilosa y $1 \mathrm{~g} / \mathrm{L}$ de furfural, la producción de biomasa disminuye $60 \%$. La descomposición de la lignina genera fenoles de bajo peso molecular, como la vainillina, la cual inhibe el crecimiento a una concentración de $2 \mathrm{~g} / \mathrm{L}$. (Zhao et al., 2012). El método de obtención del hidrolizado de lignocelulosa que genera menor concentración de compuestos inhibidores del crecimiento de microorganismos es el DMR-EH (del inglés, Deacetylation and Mechanical Refining and Enzymatic Hydrolysis), a través del cual se generan hasta $230 \mathrm{~g} / \mathrm{L}$ de azúcares monoméricos (Chen et al., 2016). El pulverizado de maíz genera una disponibilidad de lignina del $15 \%$, mientras que el tratamiento DMR-EH produce un $60 \%$, y disminuye el contenido de xilano en un $31 \%$ (Salvachúa et al., 2016). El uso de la lignocelulosa como fuente de carbono para la obtención de biodiesel representa una ventaja sobre otras industrias que también utilizan lípidos como materia prima.

A pesar de la capacidad de $R$. toruloides de integrar xilosa a su metabolismo y de tener una alta estabilidad a la inserción de genes por recombinación no homóloga, características que incrementan el interés por esta levadura, la producción de metabolitos de interés (terpenos) depende de la composición de la biomasa, del sistema de hidrólisis y del sistema de fermentación: utilizando rastrojo de maíz hidrolizado con un sistema líquido iónico colina/ $\alpha$-cetoglutarato, la cepa transformada con la bisaboleno sintasa produce $261 \mathrm{mg} / \mathrm{mL}$ de bisaboleno en un fermentador de banco (at bench scale), mientras que con un tratamiento alcalino la producción pasa a ser de $680 \mathrm{mg} / \mathrm{L}$ en un fermentador de banco alimentado por lotes de alta gravedad. Si se alimenta a la levadura únicamente con glucosa, ésta se consume en $24 \mathrm{~h}$, alcanzando una producción de bisaboleno de aproximadamente 60 $\mathrm{mg} / \mathrm{L}$. Aunque hay reportes afirmando que la levadura es capaz de integrar a su metabolismo la xilosa, la asimilación de este azúcar por la levadura es pobre durante las primeras $24 \mathrm{~h}$ y se incrementa a lo largo del tiempo (72 h), pero no se ve reflejado el destino de los carbonos en la producción del bisaboleno, que es de $70 \mathrm{mg} / \mathrm{L}$ a las $72 \mathrm{~h}$ (Yaegashi et al., 2017; Zhuang et al., 2019). Mientras que en presencia del ácido $p$-cumárico, la máxima producción es de $80 \mathrm{mg} / \mathrm{L}$ y el ácido se consume desde el inicio del cultivo, es decir, el ácido se integra al metabolismo más rápido que la xilosa, pero no tan rápido como la glucosa. Resulta interesante que al combinar las tres fuentes de carbono antes mencionadas, el consumo del ácido $p$-cumárico toma ventaja sobre la glucosa y la xilosa, alcanzando una producción entre $90-100 \mathrm{mg} / \mathrm{L}$ de bisaboleno en 4 días de cultivo (Yaegashi et al., 2017). Otro factor a considerar en la producción de terpenos como el bisaboleno es el pH del medio. Aunque $R$. toruloides crece y produce los monoterpenos y ácidos orgánicos a $\mathrm{pH}$ de 3.4, el pH óptimo de producción es de 7.4 (Zhuang et al., 2019). 
Esta levadura es flexible y las condiciones de cultivo pueden adaptarse a los requerimientos. Por ejemplo, al cultivar a la levadura en un biorreactor de $20 \mathrm{~L}$, utilizando hidrolizados de biomasa (lignocelulosa), se alcanza una producción de $2.2 \mathrm{~g} / \mathrm{L}$ de bisaboleno ( Zhao et al., 2012).

La levadura $R$. toruloides tiene mayor relevancia como microorganismo productor de monoterpenos con aplicaciones biotecnológicas. Cuando se cultiva una cepa de $R$. toruloides que expresa a la bisaboleno sintasa en un medio sintético ( $2 \%$ glucosa p/v) se producen $294 \mathrm{mg} / \mathrm{L}$ de bisaboleno. Mientras que, la expresión de la misma enzima en $S$. cerevisiae y el cultivo de la cepa bajo las mismas condiciones generan $20 \mathrm{mg} / \mathrm{L}$ del bisaboleno. Sin embargo, aunque la diferencia en la producción es clara, es importante remarcar que $R$. toruloides es una cepa oleaginosa, mientras que $S$. cerevisiae no lo es, lo cual marca una diferencia metabólica. Por otra parte, el método de transformación ATMT (Agrobacterium Tumefaciens Mediated-Transformation) modifica el número de copias del gen de la bisaboleno sintasa en $R$. toruloides, que también se asocia con un aumento en la producción del bisaboleno, y aunque $S$. cerevisiae se transforme utilizando el mismo método, no se produce un aumento de su capacidad productora de los monoterpenos, lo que ha llevado a sugerir que $R$. toruloides es el mejor modelo para la producción de monoterpenos como el bisaboleno. El precursor de los terpenos es el isómero 3-isopentenil difosfato que deriva del acetil-CoA junto con el dimetilalil pirofosfato (DAPP). Del 3-isopentenil difosfato se producen el licopeno y el $\beta$-caroteno.

Otro terpeno producido por $R$. toruloides utilizando el método ATMT es el cineol o eucaliptol. La enzima encargada de producirlo es la cineol sintasa, codificada por el gen hyg3 del género de hongos ascomicetos Hypoxylon, que proliferan en la superficie de la madera muerta. La expresión de este gen en $R$. toruloides resulta en una producción de $15.81 \mathrm{mg} / \mathrm{L}$ del monoterpeno cuando se usa un medio rico y de $27.64 \mathrm{mg} / \mathrm{L}$ cuando se cultiva la levadura en rastrojo de maíz (Shaw et al., 2015; Zhuang et al., 2019). El cineol es un monoterpeno que tiene una baja solubilidad y alta densidad de energía, su número de octano (RON) y número de octano de motor (MON) son de 99.2 y 91.0, respectivamente. Sin embargo, tiene la desventaja de tener un punto de congelación de $1{ }^{\circ} \mathrm{C}$, mientras que el del etanol es de $-114^{\circ} \mathrm{C}$ (Zhuang et al., 2019). Respecto a la acumulación de lípidos, al igual que con $Y$. lipolytica, la inserción de genes relacionados con la síntesis de lípidos resulta en un aumento en la acumulación del 49\% cuando la acetil-CoA carboxilasa y la diacilglicerol aciltransferasa se insertan al azar en el genoma de $R$. toruloides (IFO 0880). Otras enzimas que se han expresado y que incrementan la acumulación de lípidos son: $\Delta 9$-ácido graso desaturasa, $\Delta 12$ ácido graso desaturasa, generando una cepa con una capacidad de acumulación del 49\% peso seco de ácido $\alpha$-linolénico. La integración de varias copias de 3-cetoacil-CoA sintasa de diferentes plantas en $R$. toruloides CECT 13085, aumenta la producción de ácidos grasos de cadena muy larga: ácido erúcico (omega-9) y ácido nervónico (omega-9) (Pimienta et al., 2019). La producción de monoterpenos no es el único objetivo en la ingeniería de $R$. toruloides y otras levaduras como S. cerevisiae (Callari, Meier, Ravasio \& Heider, 2018). También es de interés la producción de diterpenos como el ent-kaurene, que se sintetiza a partir del GGPP, vía el intermediario ent-copalil difosfato (CDP). Este diterperno da paso a la obtención de la giberelina, una fitohormona asociada con la tolerancia al estrés en plantas y que tiene una amplia aplicación en la agricultura. A partir del ent-kaurene también es posible la síntesis de compuestos con actividad anti-inflamatoria (ácido kaurenoico), anti-cáncer (limoneno y perillyl alcohol) y propiedades antimicrobianas (thymol) (Brennan et al., 2012; Geiselman et al., 2020).

\section{Conclusiones}

La generación de biomoléculas de uso potencial en la industria alimenticia y farmacéutica es posible gracias a la ingeniería genética en modelos de estudio clásicos como $S$. cerevisiae y E. coli. Sin embargo, debido a las altas demandas de compuestos como los biocombustibles, surge la necesidad de encontrar y desarrollar técnicas de ingeniería genética para otros microorganismos, diferentes de los modelos clásicos, y que presentan ventajas metabólicas como la capacidad que tiene de acumular lípidos $Y$. lipolytica o la de acumular lípidos y carotenoides como $R$. toruloides. La producción de biocombustibles es una idea prometedora, pero quedan por resolver aspectos metabólicos y condiciones relacionadas con la capacidad de incrementar la producción de los terpenos de interés sin que estos resulten tóxicos para la célula hospedera, de tal manera que se puedan obtener grandes volúmenes de biodiesel, como con el bioetanol que se obtiene de la planta del maíz o de los residuos orgánicos producto del desecho de alimentos como los cítricos. Asimismo, es necesario aumentar la resistencia de las células al estrés producido por los compuestos fenólicos que inhiben el crecimiento celular debido a las alteraciones que provocan en las membranas biológicas, o por los cambios de $\mathrm{pH}$ interno dados por la presencia de ácidos orgánicos como el acético, que alteran el balance energético de la célula. Estos efectos varían con el proceso fermentativo empleado. Además, hay que optimizar la detoxificación, es decir, neutralizar o eliminar compuestos presentes en el sustrato que resultan tóxicos para el microorganismo empleado. También queda por resolver el destino de los subproductos de la obtención del biodiesel, por ejemplo, los residuos de la transesterificación, entre los que se encuentran: el $\mathrm{HCl}$, el $\mathrm{NaOH}$ o el $\mathrm{KOH}$, y el glicerol, compuestos altamente perjudiciales para el medio ambiente y para la salud. También hay que considerar las enormes cantidades de agua que se necesitan en la producción de biocombustibles. 


\section{Agradecimientos}

Los autores agradecemos a la Universidad Nacional Autónoma de México-Programa de Apoyo a Proyectos de Investigación (PAPIIT-IN222117 e IA200321) y al Consejo Nacional de Ciencia y Tecnología (CONACYT-254904).

\section{REFERENCIAS}

Beopoulos, A., Verbeke, J., Bordes, F., Guicherd, M., Bressy, M., Marty, A. \& Nicaud, J.-M. (2014). Metabolic engineering for ricinoleic acid production in the oleaginous yeast Yarrowia lipolytica. Applied Microbiology and Biotechnology, 98(1), 251-262. https://doi.org/10.1007/ s00253-013-5295-x

Brennan, T. C., Turner, C. D., Kromer, J. O. \& Nielsen, L. K. (2012). Alleviating monoterpene toxicity using a twophase extractive fermentation for the bioproduction of jet fuel mixtures in Saccharomyces cerevisiae. Biotechnology and Bioengineering, 109(10), 2513-2522. https://doi. org/10.1002/bit.24536

Callari, R., Meier, Y., Ravasio, D. \& Heider, H. (2018). Dynamic Control of ERG20 and ERG9 Expression for Improved Casbene Production in Saccharomyces cerevisiae. Frontiers in Bioengineering and Biotechnology, 6, 160. https://doi.org/10.3389/fbioe.2018.00160

Chang, W. C., Song, H., Liu, H. W. \& Liu, P. (2013). Current development in isoprenoid precursor biosynthesis and regulation. Current Opinion in Chemical Biology, 17(4), 571-579. https://doi.org/10.1016/j.cbpa.2013.06.020

Chen, X., Kuhn, E., Jennings, E. W., Nelson, R., Tao, L., Zhang, M. \& Tucker, M. P. (2016). DMR (deacetylation and mechanical refining) processing of corn stover achieves high monomeric sugar concentrations (230 g L-1) during enzymatic hydrolysis and high ethanol concentrations $(>10 \% \mathrm{v} / \mathrm{v})$ during fermentation without hydrolysate purification or concentration. Energy \& Environmental Science, 9(4), 1237-1245. https://doi. org/10.1039/C5EE03718B

de Paula, R. G., Antonieto, A. C. C., Ribeiro, L. F. C., Srivastava, N., O’Donovan, A., Mishra, P. K., Gupta, V. K. \& Silva, R.N (2019). Engineered microbial host selection for value-added bioproducts from lignocellulose. Biotechnology Advances, 37(6), 107347. https://doi. org/10.1016/j.biotechadv.2019.02.003

Das, M., Patra, P. \& Ghosh, A. (2020). Metabolic engineering for enhancing microbial biosynthesis of advanced biofuels. Renewable and Sustainable Energy Reviews, 119, 109562. https://doi.org/10.1016/j.rser.2019.109562

Dinh, H. V., Suthers, P. F., Chan, S. H. J., Shen, Y., Xiao, T., Deewan, A., Jagtap, S. S., Zhao, H., Ro, C. V., Rabinowitz, J. D. \& Maranas, C. D. (2019). A comprehensive genomescale model for Rhodosporidium toruloides IFO0880 accounting for functional genomics and phenotypic data. Metabolic Engineering Communications, 9, e00101. https://doi.org/10.1016/j.mec.2019.e00101
Ericsson, J., Appelkvist, E. L., Runquist, M. \& Dallner, G. (1993). Biosynthesis of dolichol and cholesterol in rat liver peroxisomes. Biochimie, 75(3-4), 167-173. https:// doi.org/10.1016/0300-9084(93)90074-3

Gajdos, P., Ledesma-Amaro, R., Nicaud, J. M., Certik, M. \& Rossignol, T. (2016). Overexpression of diacylglycerol acyltransferase in Yarrowia lipolytica affects lipid body size, number and distribution. FEMS Yeast Research., 16(6). https://doi.org/10.1093/femsyr/fow062

Geiselman, G. M., Zhuang, X., Kirby, J., Tran-Gyamfi, M. B., Prahl, J. P., Sundstrom, E. R., Gao, Y., Munoz Munoz, N., Nicora, C. D., Clay, D.M., Papa, G., BurnumJohnson, K. E., MAgnuson, J. K., Tanjore, D., Skerker, J. M. \& Gladden, J. M (2020). Production of ent-kaurene from lignocellulosic hydrolysate in Rhodosporidium toruloides. Microbial Cell Factories, 19(1), 24. https:// doi.org/10.1186/s12934-020-1293-8

George, K. W., Alonso-Gutierrez, J., Keasling, J. D. \& Lee, T. S. (2015). Isoprenoid Drugs, Biofuels, and ChemicalsArtemisinin, Farnesene, and Beyond. In J. Schrader \& J. Bohlmann (Eds.), Biotechnology of Isoprenoids (pp. 355-389). https: //doi.org/10.1007/10_2014_288

Gupta, P. \& Phulara, S. C. (2015). Metabolic engineering for isoprenoid-based biofuel production. Journal of Applied Microbiology, 119(3), 605-619. https://doi.org/10.1111/ jam.12871

Hakizimana, O., Matabaro, E. \& Lee, B. H. (2020). The current strategies and parameters for the enhanced microbial production of 2,3-butanediol. Biotechnology Reports, 25, e00397. https://doi.org/10.1016/j.btre.2019.e00397

Hu, Z., He, B., Ma, L., Sun, Y., Niu, Y. \& Zeng, B. (2017). Recent Advances in Ergosterol Biosynthesis and Regulation Mechanisms in Saccharomyces cerevisiae. Indian Journal of Microbiology, 57(3), 270-277. https:// doi.org/10.1007/s12088-017-0657-1

Imatoukene, N., Verbeke, J., Beopoulos, A., Idrissi Taghki, A., Thomasset, B., Sarde, C.-O., Nonus, M. \& Nicaud, J.-M. (2017). A metabolic engineering strategy for producing conjugated linoleic acids using the oleaginous yeast Yarrowia lipolytica. Applied Microbiology and Biotechnology, 101(11), 4605-4616. https://doi. org/10.1007/s00253-017-8240-6

Langi, P., Kiokias, S., Varzakas, T. \& Proestos, C. (2018). Carotenoids: From Plants to Food and Feed Industries. Methods in Molecular Biology, 1852, 57-71. https://doi. org/10.1007/978-1-4939-8742-9_3

Li, M., Hou, F., Wu, T., Jiang, X., Li, F., Liu, H., Xian, M. \& Zhang, H. (2020). Recent advances of metabolic engineering strategies in natural isoprenoid production using cell factories. Natural Product Reports, 37(1), 80-99. The Royal Society of Chemistry. https://doi. org/10.1039/C9NP00016J

Liu, Y., Koh, C. M. J., Yap, S. A., Du, M., Hlaing, M. M. \& Ji, L. (2018). Identification of novel genes in the 
carotenogenic and oleaginous yeast Rhodotorula toruloides through genome-wide insertional mutagenesis. BMC Microbiology, 18(1), 14. https://doi.org/10.1186/ s12866-018-1151-6

Mukhtar, Y. M., Adu-Frimpong, M., Xu, X. \& Yu, J. (2018). Biochemical significance of limonene and its metabolites: Future prospects for designing and developing highly potent anticancer drugs. Bioscience Reports, 38(6). https://doi.org/10.1042/BSR20181253

Niehus, X., Crutz-Le Coq, A. M., Sandoval, G., Nicaud, J. M. \& Ledesma-Amaro, R. (2018). Engineering Yarrowia lipolytica to enhance lipid production from lignocellulosic materials. Biotechnol for Biofuels, 11, 11. https://doi. org/10.1186/s13068-018-1010-6

Pimienta, J. A. P., Papa, G., Rodriguez, A., Barcelos, C. A., Liang, L., Stavila, V., Sanchez, A., Gladden, J. M. \& Simmons, B. A. (2019). Pilot-scale hydrothermal pretreatment and optimized saccharification enables bisabolene production from multiple feedstocks. Green Chemistry, 21(11), 3152-3164. The Royal Society of Chemistry. https://doi.org/10.1039/C9GC00323A

Pourbafrani, M., Forgacs, G., Horvath, I. S., Niklasson, C. \& Taherzadeh, M. J. (2010). Production of biofuels, limonene and pectin from citrus wastes. Bioresource Technology, 101(11), 4246-4250. https://doi. org/10.1016/j.biortech.2010.01.077

Rodriguez, A., Ersig, N., Geiselman, G. M., Seibel, K., Simmons, B. A., Magnuson, J. K., Eudes, A. \& Gladden, J. M. (2019). Conversion of depolymerized sugars and aromatics from engineered feedstocks by two oleaginous red yeasts. Bioresource Technology, 286, 121365. https:// doi.org/10.1016/j.biortech.2019.121365

Rossoni, L., Carr, R., Baxter, S., Cortis, R., Thorpe, T., Eastham, G. \& Stephens, G. (2018). Engineering Escherichia coli to grow constitutively on D-xylose using the carbon-efficient Weimberg pathway. Microbiology, 164(3), 287-298. https://doi.org/10.1099/mic.0.000611

Salis, A., Pinna, M., Monduzzi, M. \& Solinas, V. (2005). Biodiesel production from triolein and short chain alcohols through biocatalysis. Journal of Biotechnology, 119(3), 291-299. https://doi.org/10.1016/j.jbiotec.2005.04.009

Salvachúa, D., Katahira, R., Cleveland, N. S., Khanna, P., Resch, M. G., Black, B. A., Purvine, S. O.,Zink, E. M., Prieto, A., Martínez, M. J., Martínez, Angel T., Simmons, B. A., Gladden, J. M. \& Beckhmam, G. T. (2016). Lignin depolymerization by fungal secretomes and a microbial sink. Green Chemistry, 18, 6046-6062. https://doi. org/10.1039/C6GC021531J

Shaw, J. J., Berbasova, T., Sasaki, T., Jefferson-George, K., Spakowicz, D. J., Dunican, B. F., Portero, C. E., NarvaezTrujillo, A. \& Strobel, S. A. (2015). Identification of a fungal 1,8-cineole synthase from Hypoxylon sp. With specificity determinants in common with the plant synthases. Journal of Biological Chemistry, 290(13),
8511-8526. https://doi.org/10.1074/jbc.M114.636159

Singh, G., Jeyaseelan, C., Bandyopadhyay, K. K. \& Paul, D. (2018). Comparative analysis of biodiesel produced by acidic transesterification of lipid extracted from oleaginous yeast Rhodosporidium toruloides. 3 Biotechnology 8(10), 434. https://doi.org/10.1007/s13205-018-1467-9

Tetali, S. D. (2019). Terpenes and isoprenoids: A wealth of compounds for global use. Planta, 249(1), 1-8. https:// doi.org/10.1007/s00425-018-3056-X

Toivari, M. H., Salusjarvi, L., Ruohonen, L. \& Penttila, M. (2004). Endogenous xylose pathway in Saccharomyces cerevisiae. Applied Environmental Microbiology, 70(6), 3681-3686. https://doi.org/10.1128/AEM.70.6.36813686.2004

Wen, Z., Zhang, S., Odoh, C. K., Jin, M. \& Zhao, Z. K. (2020). Rhodosporidium toruloides - A potential red yeast chassis for lipids and beyond. FEMS Yeast Research, 20(5). https://doi.org/10.1093/femsyr/foaa038

Xu, P., Qiao, K., Ahn, W. S. \& Stephanopoulos, G. (2016). Engineering Yarrowia lipolytica as a platform for synthesis of drop-in transportation fuels and oleochemicals. Proceeding of the National Academic of Sciences of the United States of America, 113(39), 10848-10853. https:// doi.org/10.1073/pnas.1607295113

Yaegashi, J., Kirby, J., Ito, M., Sun, J., Dutta, T., Mirsiaghi, M., Sundstrom, E. R., Rodriguez, A., Baidoo, E., Tanjore, D., Pray, T., Sale, K., Singh, S., Keasling, J. D., Simmons, B. A Singer, S. W., Magnuson, J. K., Arkin, A. P., Skerker, J. M. \& Gladden, J. M. (2017). Rhodosporidium toruloides: A new platform organism for conversion of lignocellulose into terpene biofuels and bioproducts. Biotechnology for Biofuels, 10, 241. https://doi.org/10.1186/s13068-0170927-5

Yusoff, M. F. M., Xu, X. \& Guo, Z. (2014). Comparison of Fatty Acid Methyl and Ethyl Esters as Biodiesel Base Stock: A Review on Processing and Production Requirements. Journal of the American Oil Chemists' Society, 91(4), 525-531. https://doi.org/10.1007/s11746014-2443-0

Zada, B., Wang, C., Park, J. B., Jeong, S. H., Park, J. E., Singh, H. B. \& Kim, S. W. (2018). Metabolic engineering of Escherichia coli for production of mixed isoprenoid alcohols and their derivatives. Biotechnology for Biofuels, 11, 210. https://doi.org/10.1186/s13068-018-1210-0

Zhang, J. \& Zhao, C. (2015). A new approach for bio-jet fuel generation from palm oil and limonene in the absence of hydrogen. Chemical Communications (Camb), 51(97), 17249-17252. https://doi.org/10.1039/c5cc06601h

Zhao, J., Li, C., Zhang, Y., Shen, Y., Hou, J. \& Bao, X. (2017). Dynamic control of ERG20 expression combined with minimized endogenous downstream metabolism contributes to the improvement of geraniol production in Saccharomyces cerevisiae. Microbial Cell Factories, 16(1), 17. https://doi.org/10.1186/s12934-017-0641-9 
Zhao, X., Peng, F., Du, W., Liu, C. \& Liu, D. (2012). Effects of some inhibitors on the growth and lipid accumulation of oleaginous yeast Rhodosporidium toruloides and preparation of biodiesel by enzymatic transesterification of the lipid. Bioprocess Biosystems Engineering, 35(6), 993-1004. https://doi.org/10.1186/ s12934-017-0641-9
Zhuang, X., Kilian, O., Monroe, E., Ito, M., Tran-Gymfi, M. B., Liu, F., Davis, R. W., Mirsiaghi, M., Sundstrom, E., Pray, T., Skerker, J. M., George, A. \& Gladden, J. M. (2019). Monoterpene production by the carotenogenic yeast Rhodosporidium toruloides. Microbial Cell Factories, 18(1), 54. https://doi.org/10.1186/s12934-0191099-8 